\title{
INPP4B promotes cell survival via SGK3 activation in NPM1-mutated leukemia
}

\author{
Hongjun Jin', Liyuan Yang1', Lu Wang', Zailin Yang², Qian Zhan³, Yao Tao', Qin Zou', Yuting Tang', \\ Jingrong Xian', Shuaishuai Zhang ${ }^{1}$, Yipei Jing ${ }^{1}$ and Ling Zhang ${ }^{1 *}$
}

\begin{abstract}
Background: Acute myeloid leukemia (AML) with mutated nucleophosmin (NPM1) has been recognized as a distinct leukemia entity in the 2016 World Health Organization (WHO) classification. The genetic events underlying oncogenesis in NPM1-mutated AML that is characterized by a normal karyotype remain unclear. Inositol polyphosphate 4-phosphatase type II (INPP4B), a new factor in the phosphoinositide-3 kinase (PI3K) pathwayassociated cancers, has been recently found a clinically relevant role in AML. However, little is known about the specific mechanistic function of INPP4B in NPM1-mutated AML.

Methods: The INPP4B expression levels in NPM1-mutated AML primary blasts and AML OCI-AML3 cell lines were determined by qRT-PCR and western blotting. The effect of INPP4B knockdown on OCI-AML3 leukemia cell proliferation was evaluated, using the Cell Counting Kit-8 and colony formation assay. After INPP4B overexpression or knockdown, the activation of serum and glucocorticoid-regulated kinase 3 (SGK3) and AKT was assessed. The effects of PI3K signaling pathway inhibitors on the levels of p-SGK3 in OCl-AML3 cells were tested. The mass of PI $(3,4) P_{2}$ and PI (3) P was analyzed by ELISA upon INPP4B overexpression. Knockdown of SGK3 by RNA interference and a rescue assay were performed to confirm the critical role of SGK3 in INPP4B-mediated cell survival. In addition, the molecular mechanism underlying INPP4B expression in NPM1-mutated leukemia cells was explored. Finally, Kaplan-Meier survival analysis was conducted on the NPM1-mutated AML cohort stratified into quartiles for INPP4B expression in The Cancer Genome Atlas (TCGA) dataset.
\end{abstract}

Results: High expression of INPP4B was observed in NPM1-mutated AML. Knockdown of INPP4B repressed cell proliferation in OCI-AML3 cells, whereas recovered INPP4B rescued this inhibitory effect in vitro. Mechanically, INPP4B enhanced phosphorylated SGK3 (p-SGK3) status, but did not affect AKT activation. SGK3 was required for INPP4B-induced cell proliferation in OCI-AML3 cells. High levels of INPP4B were at least partially caused by the NPM1 mutant via ERK/Ets-1 signaling. Finally, high expression of INPP4B showed a trend towards lower overall survival and event-free survival in NPM1-mutated AML patients.

Conclusions: Our results indicate that INPP4B promotes leukemia cell survival via SGK3 activation, and INPP4B might be a potential target in the treatment of NPM1-mutated AML.

Keywords: Acute myeloid leukemia, Nucleophosmin, Gene mutation, INPP4B, SGK3, AKT, Ets-1, Cell survival

\footnotetext{
* Correspondence: lingzhang@cqmu.edu.cn

${ }^{1}$ Key Laboratory of Laboratory Medical Diagnostics Designated by the

Ministry of Education, School of Laboratory Medicine, Chongqing Medical

University, Chongqing 400016, China. No.1, Yixueyuan Road, Chongqing

400016, China

Full list of author information is available at the end of the article
} 


\section{Background}

Acute myeloid leukemia (AML) is an aggressive bone marrow malignancy that arises from the somatic transformation of hematopoietic stem and progenitor cells [1]. Cytogenetic analysis assigns about $50 \%$ of AML patients to the normal karyotype (NK-AML) [2-4]. The discovery of numerous genetic alterations that are associated with NK-AML through whole-genome analyses has led to significant advances in AML research [5]. Nucleophosmin (NPM1) is one of the most frequently altered genes in NK-AML, accounting for one-third of AML cases (50 to $60 \%$ of adult NK-AML) [6]. To date, > 55 unique mutations have been identified in exon 12 of the gene NPM1, and the most common mutation is referred to as the type A mutation (NPM1-mA) with a 4-base-pair TCTG insertion at nucleotide position 960 [7, 8]. Because of its distinctive molecular and clinical characteristics, NPM1mutated AML has been defined as a distinct entity in the 2016 updated World Health Organization (WHO) classification of myeloid neoplasms [9, 10]. Although the NPM1 mutation is an AML-driving lesion, this mutation alone is not sufficient to cause AML and it requires a cooperative event that aids leukemogenesis [11].

An accumulating body of evidence indicates that the phosphoinositide-3 kinase (PI3K) pathway plays an important role in the regulation of hematopoiesis [12]. Abnormal activation of the PI3K signaling pathway has been reported in $>50 \%$ of AML cases [13]. Activated PI3K phosphorylates the phosphatidyl-inositol bisphosphate $\left(\mathrm{PIP}_{2}\right)$ to generate phosphatidyl-inositol trisphosphate $\left(\mathrm{PIP}_{3}\right)$, and thereby facilitates recruitment and activation of the AKT protein $[14,15]$. In turn, PI3K/AKT signaling can be terminated by several phosphoinositide phosphatases that dephosphorylate phosphatidylinositol phosphate (PIP) species, i.e., the phosphatase and tensin homolog (PTEN) and src homology 2-containing inositol phosphatase (SHIP) family hydrolyze $\mathrm{PIP}_{3}$ to generate $\mathrm{PIP}_{2}[16,17]$. Recently, Inositol polyphosphate 4-phosphatase type II (INPP4B), a new factor in the regulation of the PI3K signaling module in tumors, was observed to preferentially dephosphorylate PI $(3,4) \mathrm{P}_{2}$ to produce PI (3) P and thereby block the activation of AKT $[18,19]$. The suppressive function of INPP4B, akin to that of PTEN, was initially identified in breast cancer [20], and later confirmed in ovarian [21] and prostate cancers [22]. Interestingly, several recent reports have shown that INPP4B overexpression could be detected in other cancer contexts, such as PIK3CA-mutant breast cancer [23] and a subset of melanoma [24]. Notably, INPP4B was aberrantly overexpressed and emerged as an independent predictor of poor prognosis in AML patients with normal cytogenetics [25]. To date, however, the biological role of INPP4B in NPM1-mutated AML and the molecular mechanisms by which INPP4B contributes to leukemogenesis remain unclear.
In breast and other epithelial cancers, INPP4B has been predicted to be a tumor suppressor that blocks AKT activation. However, INPP4B expression is not associated with the changes in AKT phosphorylation status in leukemia, indicating that AKT-independent mechanisms are likely at play [25]. Serum and glucocorticoid-regulated kinase-3 (SGK3), another PI3K-dependent serine/threonine kinase, shares high structural and functional similarities with the AKT protein [26]. However, unlike AKT, SGK3 contains a unique N-terminal phox homology (PX) domain that binds to PI (3) P, thus targeting early endosomes where SGK3 is fully activated $[27,28]$. Indeed, SGK3 emerges as an alternative downstream effector of INPP4B that diverges from canonical AKT signaling [29]. Recent studies have indicated an association between high INPP4B expression and SGK3 phosphorylation levels in PIK3CA-mutant breast cancers and melanoma, in which INPP4B-mediated activation of SGK3 enhances cell proliferation and promotes anchorage-independent cell growth [23, 24]. Herein, we report that INPP4B is frequently upregulated in NPM1mutated AML, and promotes leukemia cell survival in a SGK3-dependent and AKT-independent manner. Increased INPP4B expression is partially caused by the NPM1 mutant through ERK/Ets-1 signaling. In addition, high INPP4B is associated with poor outcome in NPM1mutated patients in our study. Previous reports and the present study suggest that INPP4B provides a survival advantage through the activation of SGK3 in NPM1mutated leukemia cells. These findings further indicate that INPP4B might be a potential target for the treatment of NPM1-mutated AML.

\section{Methods}

\section{The cancer genome atlas (TCGA) gene expression data analysis}

Gene expression levels and clinical information of 200 AML patients were retrieved from The Cancer Genome Atlas (TCGA, http://www.cancergenome.nih.gov). A total of 171 samples had IlluminaGA RNA-Seq gene expression data. Clinical data and INPP4B mRNA expression data for AML samples were analyzed using the cBioPortal for Cancer Genomics. The INPP4B mRNA expression was compared between AML cases with the NPM1 mutation $(n=41)$ and those without the NPM1 mutation $(n=130)$.

\section{Patient samples}

Peripheral blood samples of 36 AML patients, who had been recently diagnosed, including 22 NPM1unmutated and 14 NPM1-mutated cases, were obtained from Southwest Hospital of the Third Military Medical University and the First Affiliated Hospital of Chongqing Medical University. Mononuclear cells were enriched by Ficoll gradient purification. The isolated mononuclear cells were used for analyses of NPM1-mA 
and INPP4B relative expression. Details of the Clinical characteristics of patients are provided in Table 1 .

\section{Cell cultures}

Human myeloid leukemia cells HL60, KG1a, K562 and THP-1 were obtained from the American Type Culture Collection (ATCC, MD, USA). The OCI-AML3 AML cells harboring NPM1-mA [30] were obtained from Deutsche Sammlung von Mikroorganismen und Zellkulturen GmbH (DSMZ, Braunschweig, Germany). All cell lines were routinely cultured in RPMI 1640 medium (Gibco, MD, USA), supplemented with $10 \%$ fetal bovine serum (FBS; Gibco, MD, USA) and 1\%

Table 1 Clinical Characteristics of Newly Diagnosed AML Patients

\begin{tabular}{|c|c|c|}
\hline Characteristics & Median (range) & No. of cases \\
\hline \multicolumn{3}{|l|}{ Sex } \\
\hline Female & & 19 \\
\hline Male & & 17 \\
\hline Total & & 36 \\
\hline \multicolumn{3}{|l|}{ Age } \\
\hline Median, years & $53.8(26-79)$ & \\
\hline \multicolumn{3}{|l|}{$W B C$} \\
\hline Median, $\times 10^{9} / \mathrm{L}$ & $44(0.3-295.0)$ & \\
\hline \multicolumn{3}{|l|}{ Platelets } \\
\hline Median, $\times 10^{9} / \mathrm{L}$ & $57.3(3.0-655.0)$ & \\
\hline \multicolumn{3}{|l|}{ AML FAB subtype } \\
\hline AML without maturation: M1 & & 4 \\
\hline AML with maturation: M2 & & 6 \\
\hline Acute promyelocytic leukemia: M3 & & 9 \\
\hline Acute myelomonocytic leukemia: M4 & & 7 \\
\hline $\begin{array}{l}\text { Acute monoblastic or monocytic } \\
\text { leukemia: M5 }\end{array}$ & & 9 \\
\hline Other subtype & & 1 \\
\hline \multicolumn{3}{|l|}{ Karyotype } \\
\hline Normal & & 14 \\
\hline $\mathrm{t}(8 ; 21)$ & & 5 \\
\hline$t(15 ; 17)$ & & 6 \\
\hline inv. (16) & & 7 \\
\hline Unknown & & 4 \\
\hline \multicolumn{3}{|l|}{ Gene mutations } \\
\hline NPM1 & & 14 \\
\hline FLT3-ITD & & 10 \\
\hline WT1 & & 9 \\
\hline CBFB-MYH11 & & 5 \\
\hline
\end{tabular}

Abbreviations: $A M L$ acute myeloid leukemia, WBC white blood cell; FAB classification, French-American-British classification, a classification of acute leukemia produced by three-nation joint collaboration penicillin and streptomycin (Beyotime, Shanghai, China) in a $5 \% \mathrm{CO}_{2}$ humidified incubator at $37^{\circ} \mathrm{C}$.

Reverse transcription PCR and quantitative real-time PCR Total RNA was isolated using the TRIzol reagent (Takara, Kyoto, Japan), and transcribed into cDNA using the PrimeScript $^{\text {tw }}$ RT Reagent Kit (Takara, Kyoto, Japan). Quantitative real-time PCR (qRT-PCR) analysis was performed on an MJ Mini ${ }^{\text {Tu }}$ Gradient Thermal Cycler Real-Time PCR machine (Bio-Rad, CA, USA) with the SYBR Green reaction kit (KAPA Biosystems, MA, USA). The following primers were used for real-time amplification: $I N P P 4 B$ (Forward 5'-GGAAAGTGTGAGCGGAAAAG-3' and Reverse 5' - CGAATTCGCATCCACTTATTG-3'); NPM1$m A$ (Forward F: 5'-TGGAGGTGGTAGCAAGGTTC-3' and Reverse 5'-CTTCCTCC ACTGCCAGACAGA-3'); SGK3 (Forward 5'-CTGAGATCTCACCATGCAAA GAG ATCACACC-3' and Reverse 5'-GGGGCTAGCTCACAA AAATAAG TCTTCT-3'); Ets-1(Forward 5' -GTCGTGGT AAACTCGG-3' and Reverse 5'-CAG CAGGAATGACA GG-3'); $\beta$-actin (Forward 5'-TAGTTGCGTTACACCCTT TC TTG-3' and Reverse 5'-TGCTGTCACCTTCA CCGT TC-3'). The mRNA expression levels were analyzed using the $2^{-\Delta \Delta C t}$ method and expressed as a fold change.

\section{Western blotting}

The cultured cells were washed and lysed in cell extraction buffer. Equal amounts of extracts were loaded into sodium dodecyl sulfate (SDS) polyacrylamide gels for electrophoresis and transferred onto polyvinylidene difluoride (PVDF) membranes. The membranes were blocked in 5\% low-fat dry milk for $3 \mathrm{~h}$, and then incubated overnight at $4{ }^{\circ} \mathrm{C}$ with primary antibodies against INPP4B, p-SGK3 ${ }^{\mathrm{T} 320}$, SGK3, p-AKT ${ }^{\mathrm{T} 308}$, AKT, p-ERK, ERK (Cell Signaling Technology, MA, USA); p-Ets-1, Ets-1, Flag (Bioworld Technology Inc. MN, USA); NPM1-mA (Abcam, Cambrige, UK) and $\beta$-actin (Santa Cruz Biotechnology Inc. CA, USA) as loading control. Membranes were washed in Tris-buffered saline (TBS) $(10 \mathrm{mM}$ Tris$\mathrm{HCl} \mathrm{pH} \mathrm{8,150} \mathrm{mM} \mathrm{NaCl)} \mathrm{containing} 0.1 \%$ Tween 20, and then incubated with HRP-conjugated secondary antibody for $1 \mathrm{~h}$, and subsequently exposed to enhanced chemiluminescence substrate (Millipore, MA, USA). Membrane blot signals were detected using the Bio-Rad Gel Imaging System on cool image workstation II (Viagene, FL, USA). Quantification of protein expression was normalized against the $\beta$-actin protein expression using imaging software.

\section{Delivery of siRNA and cell transfection}

The siRNA targeting INPP4B, SGK3, Ets-1 and control siRNA were purchased from Genechem (Shanghai, China). The OCI-AML3 cells were transfected with siRNA using the Rfect ${ }^{\mathrm{PM}}$ siRNA Transfection Reagent (BaiDai, 
Changzhou, China) according to the manufacturer's instructions. After $48 \mathrm{~h}$ of transfection, the cells were collected for qRT-PCR or western blotting analysis. The sequences of siRNA were as follows: siINPP4B1 (sense: 5'-CCAGGAGGCAUUCUUAAGATT-3'; antisense: 5'UCUUAAGAAUGCCUCCUGGTT-3'); siINPP4B2 (sen se: 5'-GCCGCAAACUGAAUGGUAUTT-3'; antisense: 5'-AUACCAUUCAGUUUGCGGCTT-3'); siSGK3 (sen se: 5'-GCAGGACUAAACGAAUUCATT-3'; antisense: 5'-UGAAUUCGUUUA GUCCUGCTT-3'); siEts-1 (sen se: 5'-ACUUGCUACCAUCCCGUAC-3'; antisense: 5'GUACGGGAUGGUAGCAAGU-3'); Control (sense: 5'-UUCUUCGAACGUGUCACGUTT-3'; antisense: 5'ACGUGACACGUUCGGAGAATT-3').

\section{Lentiviral vectors and cell infection}

The lentivirus-based short hairpin RNA (shRNA) vectors targeting INPP4B (5'-CCATCTGAGTATCCCATCTAT-3') and scramble lentiviral vectors were purchased from Genechem (Shanghai, China). The OCI-AML3 cells were infected with lentivirus for $48 \mathrm{~h}$ in the presence of $5 \mu \mathrm{g} / \mathrm{mL}$ polybrene (Sigma, CA, USA), after which they were subjected to $2 \mu \mathrm{g} / \mathrm{mL}$ puromycin selection for $7 \mathrm{~d}$ (Sigma, CA, USA). The puromycin-resistant cells were isolated and propagated for further analysis.

\section{Plasmids and cell transfection}

The pEAK-Flag/INPP4B and pCMV-Flag/SGK3 plasmids were purchased from Addgene (http://www.addgene.org). The pEGFPC1-NPM1-mA, pEGFPC1-NPM1-wt and empty pEGFPC1 were kindly provided by Dr. Falini B (Institute of Hematology, University of Perugia, Perugia, Italy). All transfection experiments were performed using the $\mathrm{xfect}^{\mathrm{TM}}$ reagent (Clontech, CA, USA) according to the manufacturer's instructions. After $48 \mathrm{~h}$ of transfection, the cells were collected for qRT-PCR or western blotting analysis.

\section{Cell viability assay}

Cell viability was determined by the Cell Counting Kit- 8 (CCK8, Dojindo Laboratories, Japan), according to the manufacturer's instructions. Cells were seeded into 96well plates (Corning, NY, USA) in triplicate at a density of $5 \times 10^{3}$ cells per well with RPMI-1640 containing $10 \%$ FBS. The cell numbers were quantified at the indicated time points with the CCK $8\left(10 \mu \mathrm{l} /\right.$ well at $37{ }^{\circ} \mathrm{C}$ for $\left.2 \mathrm{~h}\right)$, and the numbers of cells per well were determined by measuring absorbance at $450 \mathrm{~nm}$ using the microplate reader (Eon, BioTeck, CA, USA). The cell growth curves were plotted with the cell number values as the ordinate and time as the abscissa. Each experiment was performed in triplicate.

\section{Colony formation assay}

A methylcellulose clonogenic assay was carried out to determine cell colony forming ability, by planting $1 \times 10^{3}$ cells per well in triplicate in a 24 well-plate, and maintaining those cells in RPMI 1640 medium containing 20\% FBS at $37{ }^{\circ} \mathrm{C}$ in an incubator. Colony numbers were scored 10 $\mathrm{d}$ later. The colony forming units were counted using an inverted microscope.

\section{Inhibitor treatment}

The PI3K inhibitor, LY294002 (30 $\mu \mathrm{M})$; the AKT selective inhibitor, MK-2206 (5 $\mu \mathrm{M})$; and the mTOR inhibitor, rapamycin $(5 \mu \mathrm{M})$ were used to treat OCI-AML3 cells for $24 \mathrm{~h}$ and the treated cells were harvested for western blotting. The ERK inhibitor PD98059 was used to treat OCI-AML3 cells with different concentrations $(0,10,20$ and $40 \mu \mathrm{M}$ ) for $24 \mathrm{~h}$ and the treated cells were harvested for qRT-PCR and western blotting. These inhibitors were purchased from Selleck Chemicals (Selleckchem, TX, USA).

\section{$\mathrm{PI}(3,4) \mathrm{P}_{2}$ and $\mathrm{PI}(3) \mathrm{P}$ enzyme-linked immunosorbent assay (ELISA)}

Cellular PI $(3,4) \mathrm{P}_{2}$ and PI (3) P were quantitated using RY-02853 Human PI $(3,4) P_{2}$ ELISA kit and RY-02851 Human PI (3) P ELISA kit, respectively, obtained from Runyu Biotechnology (Shanghai, China), according to the manufacturer's instruction. The results were recorded and analyzed using the microplate reader (Eon, BioTeck, CA, USA).

\section{Survival analysis}

Gene expression levels and clinical survival information of 153 AML patients, including 38 patients harboring NPM1 mutations, were retrieved from TCGA dataset. All patients were stratified by INPP4B expression levels into quartiles, to categorize patients into either a high cohort or low cohort. Kaplan-Meier data of AML patients and NPM1-mutated patients were used to analyze the overall survival (OS) and the three-year event free survival (EFS). Details of the clinical characteristics according to high or low INPP4B expression among NPM1-mutated patients from TCGA dataset are provided in Table 2.

\section{Statistical analysis}

All data were derived from three independent experiments and the results were summarized and represented as mean \pm s.d. Statistical analysis was performed, using the SPSS (Version 17.0) and GraphPad (Prism 5.0) software programs. The statistical significance of differences between each group was analyzed using the unpaired Students' $t$-test. The Kaplan-Meier survival data were analyzed using the long-rank test. Any $p$-value $<0.05$ was considered statistically significant. 
Table 2 Clinical Characteristics of NPM1-mutated AML Patients with Low or High INPP4B Expression

\begin{tabular}{|c|c|c|}
\hline Characteristics & Low INPP4B $(n=29)$ & High INPP4B $(n=9)$ \\
\hline \multicolumn{3}{|l|}{$\overline{\operatorname{sex}}$} \\
\hline Female & 15 & 6 \\
\hline Male & 14 & 3 \\
\hline \multicolumn{3}{|l|}{ Age, years } \\
\hline Median, range & $52.93(21-81)$ & $50.56(21-82)$ \\
\hline \multicolumn{3}{|l|}{$W B C, \times 10^{9} / L$} \\
\hline Median, range & $61.58(5-137)$ & $49.78(1-134)$ \\
\hline \multicolumn{3}{|l|}{ Platelets, $\times 10^{9} / \mathrm{L}$} \\
\hline Median, range & $53.86(8-174)$ & $63.56(11-232)$ \\
\hline \multicolumn{3}{|c|}{ Bone marrow blast, $\%$} \\
\hline Median, range & 78.93 (48-98) & $70.11(41-95)$ \\
\hline \multicolumn{3}{|l|}{ FAB classification } \\
\hline MO & 0 & 1 \\
\hline M1 & 11 & 2 \\
\hline M2 & 3 & 4 \\
\hline M3 & 0 & 0 \\
\hline M4 & 10 & 0 \\
\hline M5 & 5 & 2 \\
\hline M6 & 0 & 0 \\
\hline M7 & 0 & 0 \\
\hline \multicolumn{3}{|l|}{ Cytogenetics } \\
\hline Normal & 27 & 7 \\
\hline Abnormal & 0 & 1 \\
\hline Unknown & 2 & 1 \\
\hline \multicolumn{3}{|l|}{ Cytogenetic risk } \\
\hline Favorable & 0 & 0 \\
\hline Intermediate & 28 & 7 \\
\hline Adverse & 0 & 1 \\
\hline Unknown & 1 & 1 \\
\hline \multicolumn{3}{|l|}{ Gene mutations } \\
\hline FLT3-ITD & 16 & 6 \\
\hline IDHI & 8 & 0 \\
\hline
\end{tabular}

Abbreviations: $A M L$ acute myeloid leukemia, WBC white blood cell, $F A B$ classification, French-American-British classification, a classification of acute leukemia produced by three-nation joint collaboration

\section{Results}

INPP4B is highly expressed in leukemia with the NPM1 mutation

To determine the expression levels of INPP4B in AML with the NPM1 mutation, we first analyzed the publicly available TCGA RNA-seq dataset of 171 AML patients. The results showed that the INPP $4 B$ transcript levels were increased among the cases of AML with the NPM1 mutation, as compared to those without the NPM1 mutation ( $p=0.0291$, Fig. 1a). Consistent with these findings, we examined the mRNA levels of INPP4B by qRT-PCR from 36 primary AML blasts and found that INPP4B was more highly expressed in NPM1-mutated AML $(n=14)$, as compared to NPM1-unmutated AML $(n=22)$ cases $(p=0.0246$, Fig. 1b). To confirm our theory, we then assessed INPP4B mRNA and protein abundance across a panel of human myeloid leukemia cell lines. The INPP4B mRNA levels were readily detected in all but two cell lines (HL60 and KG1a) at various levels. Interestingly, the OCI-AML3 cells that naturally carried NPM1$\mathrm{mA}$, showed increased INPP4B mRNA expression (Fig. 1c). Similarly, INPP4B protein levels were also increased in OCI-AML3 cells (Fig. 1d). These results demonstrate that INPP4B showed relatively high expression in NPM1-mutated AML.

\section{INPP4B promotes cell proliferation in OCI-AML3 cells}

To evaluate the functional significance of INPP4B upregulation on cell proliferation in NPM1-mutated leukemia, OCI-AML3 cells were transfected with two individual siRNAs to silence INPP4B. Once the decreased levels of siRNA-mediated INPP4B mRNA and protein were successfully confirmed in OCI-AML3 cells (Fig. 2a and b), we found that the loss of INPP4B significantly inhibited cell proliferation in vitro (Fig. 2c). In addition, INPP4B silencing markedly reduced the number of colonies, as evidenced by a significant reduction in colony forming potential, in comparison to the controls (Fig. 2d).

We further verified the effects of INPP4B on OCIAML3 cell proliferation through a rescue experiment. First, a lentiviral vector was used to establish a stable cell line with shRNA-mediated knockdown of INPP4B, and INPP4B protein was successfully deleted in OCI-AML3 cells (Fig. 2e). The pEAK-Flag/INPP4B plasmids were then introduced into INPP4B-silenced OCI-AML3 cells and the INPP4B protein was successfully recovered (Fig. 2e). Notably, forced expression of INPP4B could reverse the inhibitory effects of INPP $4 B$ knockdown on cell proliferation (Fig. 2f). Taken together, our data indicate that INPP4B was, at least in part, required for cell proliferation in OCI-AML3 leukemia cells.

\section{INPP4B mediates SGK3 activation in OCI-AML3 cells}

To explore the potential mechanisms by which INPP4B contributes to cell proliferation in NPM1-mutated leukemia, we performed siRNA-mediated knockdown of INPP4B in OCI-AML3 cells, and the phosphorylation (activation) of SGK3 and AKT was then monitored. Our results revealed that INPP4B depletion caused a significant reduction in SGK3 phosphorylation levels, but did not affect AKT activation in OCI-AML3 cells (Fig. 3a). To confirm this notion even further, the pEAK-Flag/ INPP4B plasmids were transfected into two cell lines and INPP4B mRNA levels were increased (Fig. 3b). As 

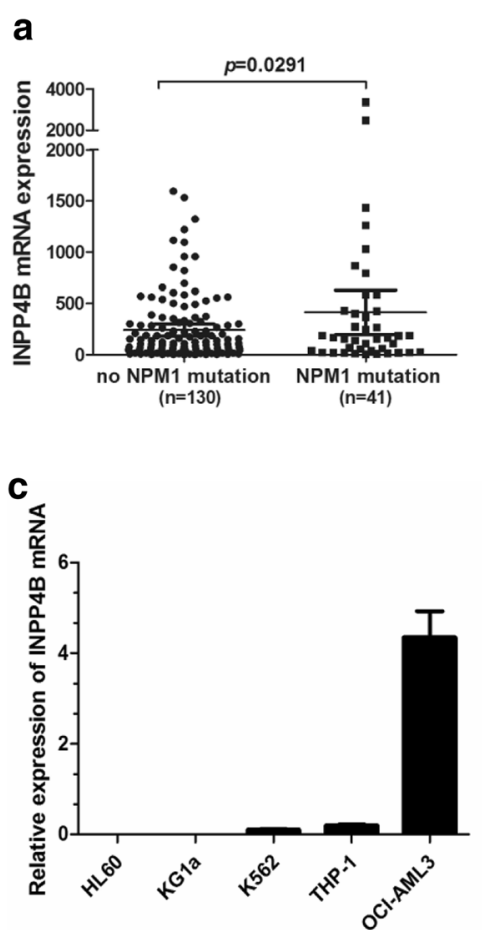

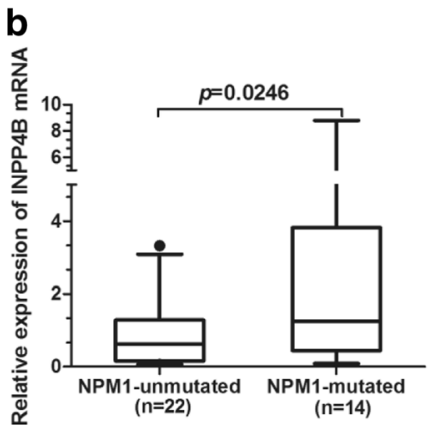

d

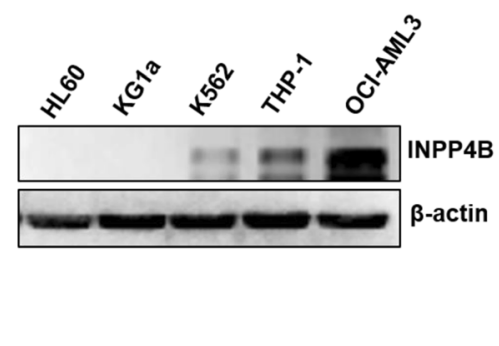

Fig. 1 High expression levels of INPP4B in leukemia cells with the NPM1 mutation. a RNA-seq mRNA expression data from the TCGA database were used to compare INPP4B expression between AML patients with $(n=41)$ and without NPM1 mutation $(n=130)$. ${ }^{*} p<0.05$. $\mathbf{b}$ The mRNA levels of INPP4B in primary NPM1-mutated AML cases $(n=14)$ were assessed by qRT-PCR, and compared to NPM1-unmutated AML cases $(n=22)$. c qRT-PCR analysis of INPP4B mRNA expression from the indicated myeloid leukemia cell lines. $\mathbf{d}$ Western blotting analysis of INPP4B and $\beta$-actin (as a loading control). Data were represented as mean \pm s.d. of three individual experiments

expected, INPP4B overexpression led to significantly elevated $\mathrm{p}-\mathrm{SGK} 3^{\mathrm{T} 320}$ levels in OCI-AML3 cells, but did not affect p-AKT ${ }^{\mathrm{T} 308}$ levels (Fig. 3c). Similar results were observed in THP-1 leukemia cells (Fig. 3d). Furthermore, the effects of the PI3K signaling pathway inhibitors on the levels of p-SGK3 in OCI-AML3 cells were tested. As shown in Fig. 3e, treatment with the PI3K inhibitor, LY294002, evidently reduced p-SGK3 levels; however, no obvious reduction in p-SGK3 levels was observed in the groups treated with the AKT-selective inhibitor, MK2206 and the mTOR inhibitor, rapamycin. Previous studies verified that the activation of SGK3 would occur at sites of PI (3) P accumulation [31]. We thus tested the impact of INPP4B on cellular levels of PI $(3,4) \mathrm{P}_{2}$ and PI (3) P. The results showed that introduction of exogenous INPP4B decreased PI $(3,4) \mathrm{P}_{2}$ levels and increased PI (3) P levels in leukemia cells (Fig. 3f, g). Collectively, our results indicate that INPP4B mediated activation of the PI3K downstream factor, SGK3, but not AKT, in OCI-AML3 cells.

\section{SGK3 is required for INPP4B-induced cell proliferation in OCl-AML3 cells}

Based on the foregoing data, we hypothesized that SGK3 might provide an advantage for INPP4B-induced cell proliferation in NPM1-mutated leukemia. To test this, we transfected the siRNA targeting SGK3 into OCIAML3 cells and found that SGK3 mRNA and protein levels were indeed reduced (Fig. 4a and b). Notably, SGK3 depletion markedly repressed cell proliferation in OCI-AML3 cells (Fig. 4c). A rescue assay was then used to verify the effects of SGK3 on INPP4B-induced cell proliferation. The results showed that reduced SGK3 phosphorylation induced by INPP4B silencing was rescued by the introduction of exogenous SGK3 (Fig. 4d). Furthermore, SGK3 overexpression partially neutralized the inhibitory effect of shINPP4B-induced cell proliferation (Fig. 4e). These results suggest that SGK3 was required for INPP4B-induced cell proliferation in OCIAML3 cells.

\section{INPP4B is upregulated by NPM1-mA via ERK/Ets-1} signaling in leukemia cells

We evaluated the potential molecular mechanism underlying INPP4B upregulation in leukemia cells with NPM1-mA. First, stably NPM1-silenced OCI-AML3 cells, for which treatment with shRNA targeting NPM1 depleted both NPM1-wt and NPM1-mA levels, were established according to previously described protocols [32] and used for further analysis. We found that the 


\section{a}

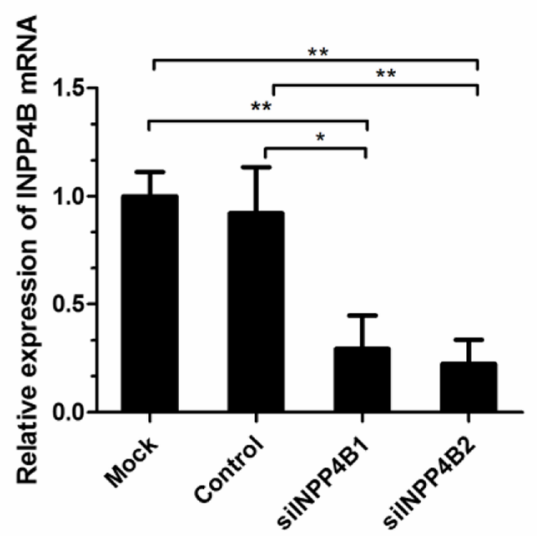

C

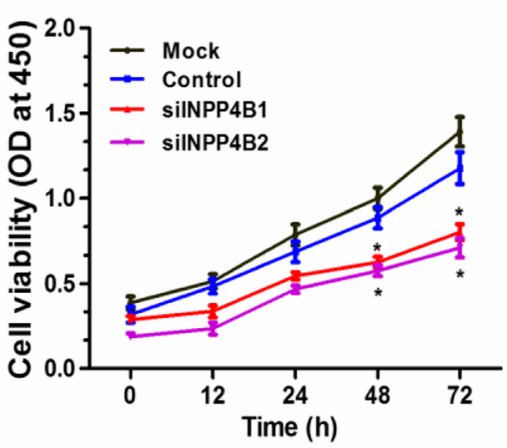

e

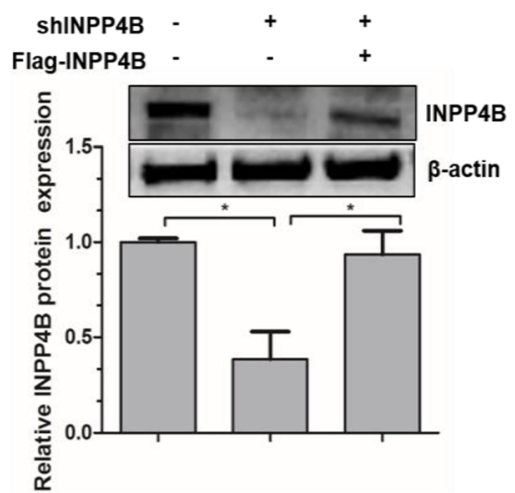

b

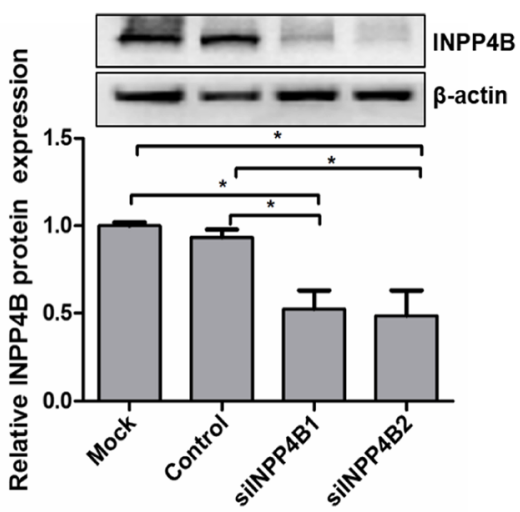

d
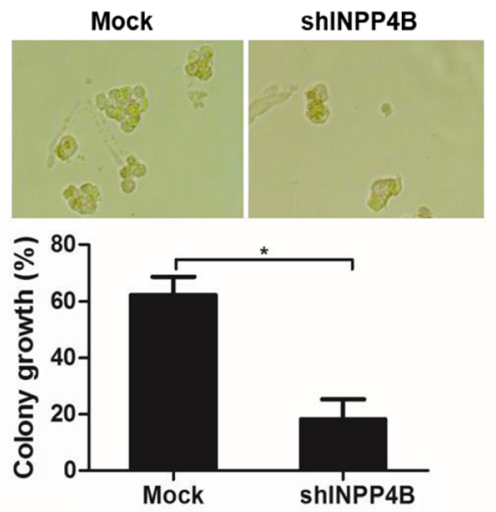

f

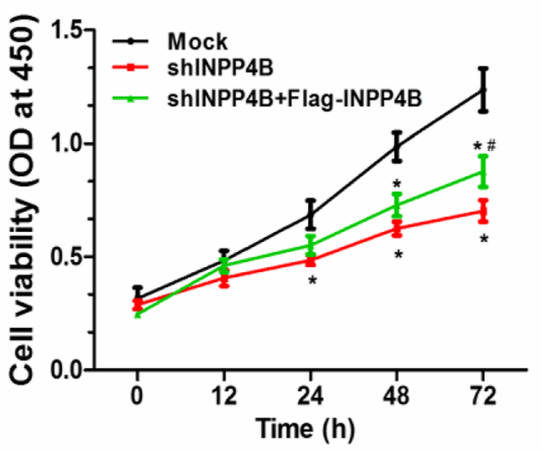

Fig. 2 INPP4B promotes cell proliferation in OCI-AML3 cells. (a) qRT-PCR and (b) western blotting analysis of INPP4B expression from the OCI-AML3 cells transduced with the control siRNA or silNPP4B. c CCK-8 assay analysis of cell proliferation activity in the silNPP4B transduced cells. d The OCI-AML3 cells stably infected with shRNA lentivirus targeting INPP4B were subjected to colony forming assays. e The INPP4B-silenced OCI-AML3 cells were transfected with the PEAK-Flag/INPP4B plasmids, western blotting analysis of INPP4B protein levels and quantified using image software normalized against $\beta$-actin. $\mathbf{f}$ CCK-8 assay analysis of cell proliferation in INPP4B-silenced OCI-AML3 cells, followed by Flag-INPP4B introduction. Data were represented as mean \pm s.d. of three individual experiments. ${ }^{*} p<0.05,{ }^{*} p<0.01$, as comparison to mock group; $\# p<0.05$, as comparison to shINPP4B group

loss of NPM1-mA significantly reduced INPP4B mRNA levels (Fig. 5a). Moreover, INPP4B protein levels and downstream phosphorylated SGK3 were considerably reduced in the NPM1-mA silenced group (Fig. 5b). This hypothesis was further confirmed, as we found that INPP $4 B$ mRNA levels were increased upon ectopic overexpression of NPM1-mA in THP-1 and K562 leukemia cell lines (Fig. 5c). Moreover, INPP4B and phosphorylated SGK3 proteins were consistently elevated, due to the introduction of NPM1-mA (Fig. 5d). This suggests that upregulation of INPP4B/SGK3 was at least partially caused by NPM1-mA expression in leukemia cells. 
a

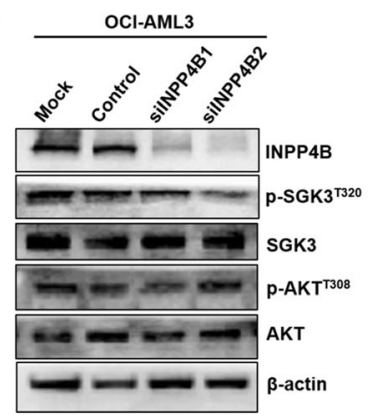

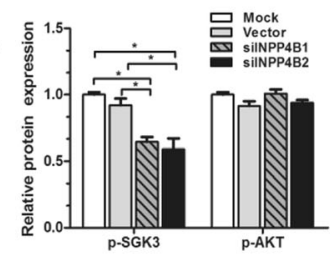

b

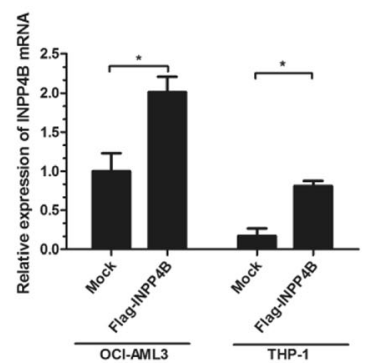

C
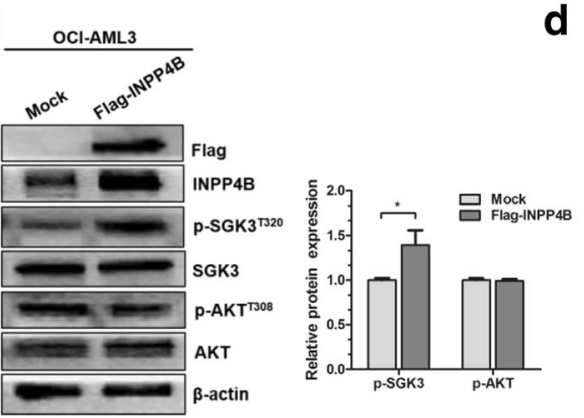

d
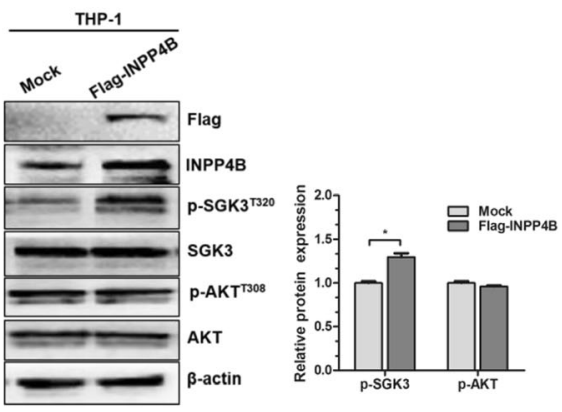

e

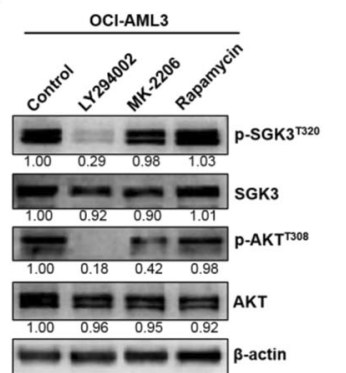

f

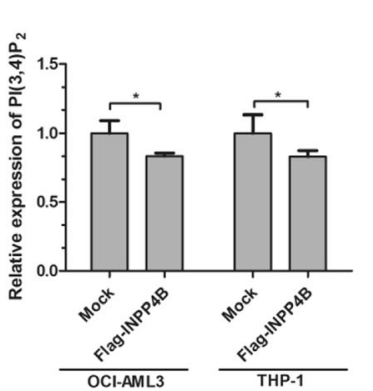

g

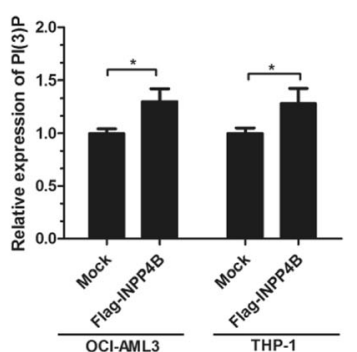

Fig. 3 INPP4B mediates SGK3 activation in OCl-AML3 cells. a Western blotting analysis of INPP4B, p-SGK3 ${ }^{T 320}$, SGK3, p-AKT ${ }^{\text {T308 }}$ and AKT from OCI-AML3 cells transduced with the control siRNA or silNPP4B. $\mathbf{b}$ qRT-PCR analysis of INPP4B mRNA expression from OCI-AML3 and THP-1 cells transduced with the pEAKFlag/INPP4B plasmids. c-d Western blotting analysis of Flag, INPP4B, p-SGK3 ${ }^{T 320}$, SGK3, p-AKT ${ }^{T 308}$ and AKT from the INPP4B transduced cells. e Western blotting analysis of p-SGK3 ${ }^{\text {T320 }}$, SGK3, p-AKT ${ }^{\text {T308 }}$ and AKT from the OCI-AML3 cells treated with LY294002 $(30 \mu M)$, MK-2206 (5 $\mu$ M) and Rapamycin (5 $\mu$ M), respectively. Proteins were quantified using image software and normalized against $\beta$-actin. The relative abundance of $(\mathbf{f}) \mathrm{Pl}(3,4) \mathrm{P} 2$ and $(\mathbf{g}) \mathrm{PI}(3) \mathrm{P}$ in the INPP4B transduced cells was measured using ELISA. Data were represented as mean \pm s.d. of three individual experiments. ${ }^{*} p<0.05$

A recent study demonstrated that the increase in INPP4B is due to Ets-1-mediated transcriptional upregulation in colon cancer cells [33]. To test this effect in NPM1-mutated AML, the siRNA targeting Ets-1 was transfected into OCI-AML3 cells, and Ets-1 knockdown was found to reduce INPP4B mRNA expression significantly (Fig. 5e). In addition, deletion of Ets-1 evidently downregulated INPP4B protein levels (Fig. 5f). Given that Ets-1 could be phosphorylated by ERK signaling, and thereby enhanced its transactivation [34], we thus treated the OCI-AML3 cells with the ERK inhibitor (PD98059) and observed that the levels of p-Ets-1 were decreased in a dose-dependent manner (Fig. 5g). Meanwhile, the INPP4B protein and mRNA levels were considerably reduced (Fig. $5 \mathrm{~g}$ and h). We previously determined that the ERK activation was continuously increased by NPM1-mA in leukemia cells [35]. Next, we observed that NPM1-mA knockdown impaired ERK/Ets-1 signaling and further reduced INPP4B protein levels in OCI-AML3 cells (Fig. 5i). Taken together, these findings indicate that INPP4B expression might be upregulated by NPM1-mA via ERK/Ets-1 signaling in leukemia cells.

\section{NPM1-mA-mediated INPP4B upregulation promotes cell proliferation in $\mathrm{OCI}-\mathrm{AML} 3$ cells}

Given that NPM1-mA enhanced INPP4B expression, we then validated the impact of NPM1-mA on the proliferation of leukemia cells. The results showed that knockdown 


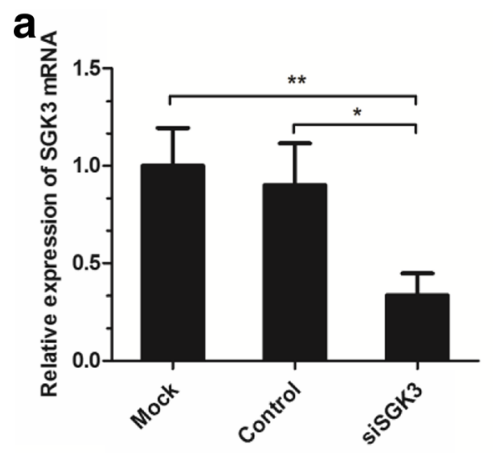

b
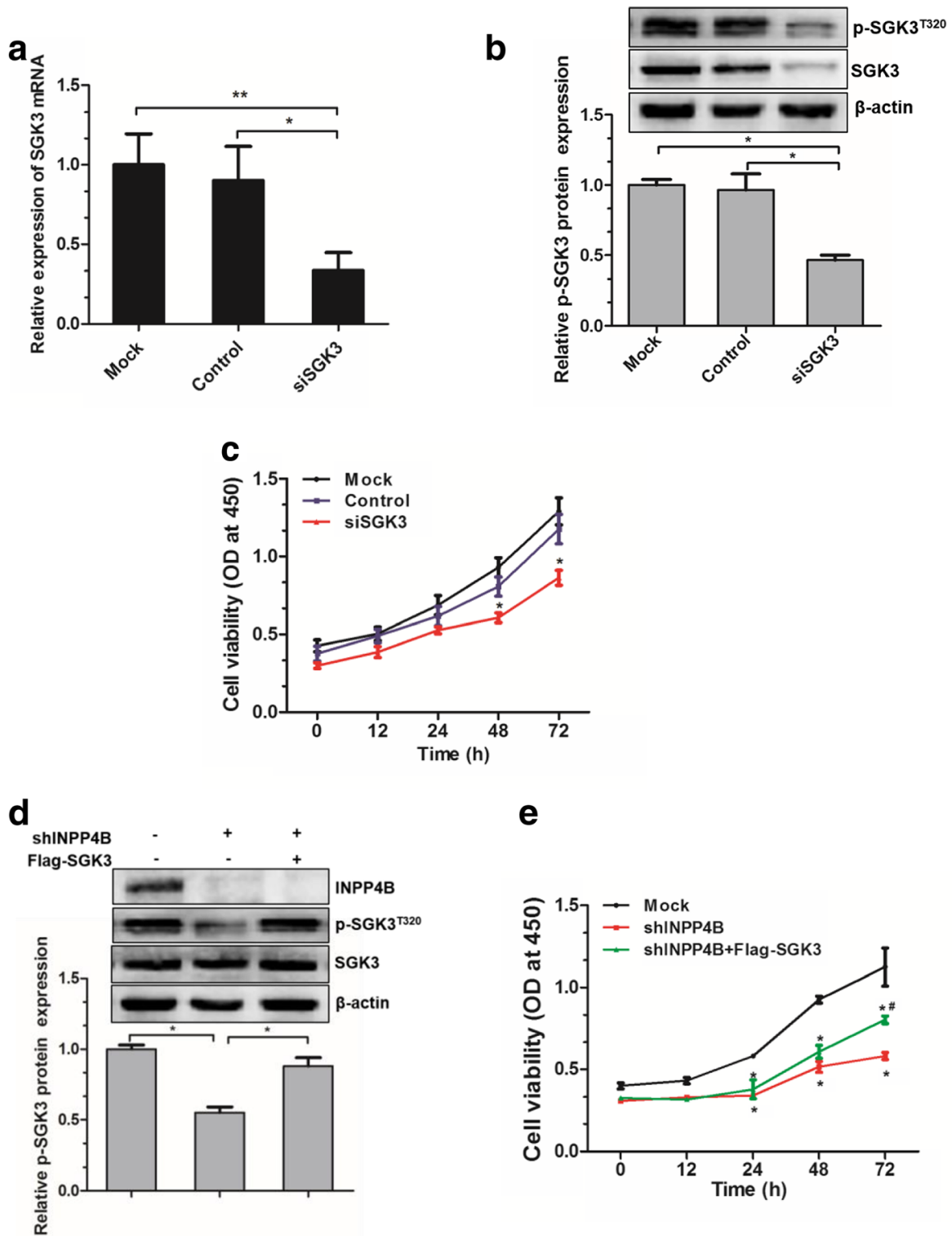

Fig. 4 SGK3 is required for INPP4B-induced cell proliferation in OCI-AML3 cells. qRT-PCR (a) and western blotting (b) analysis of SGK3 expression from the OCI-AML3 cells transduced with the control siRNA or siSGK3. c CCK-8 assay analysis of cell proliferation activity in the siSGK3 transduced cells. $\mathbf{d}$ The INPP4B-silenced OCI-AML3 cells were transfected with the pCMV-Flag/SGK3 plasmids, western blotting analysis of SGK3, p-SGK3 ${ }^{T 320}$ and INPP4B. Proteins were quantified using image software and normalized against $\beta$-actin. e CCK-8 assay analysis of cell proliferation in INPP4Bsilenced OCI-AML3 cells, followed by Flag-SGK3 introduction. Data were represented as mean \pm s.d. of three individual experiments. ${ }^{*} p<0.05$, as comparison to mock or control group, respectively; $\# p<0.05$, as comparison to shINPP4B group

of NPM1-mA significantly attenuated cell proliferation in OCI-AML3 cells, as compared to the control group (Fig. 6a). In addition, the colony formation assay showed a decreased colony size and a lower proportion of colony forming units in the NPM1-mA-silenced OCI-AML3 cells (Fig. 6b). To further evaluate the pivotal role of NPM1-mA mediated INPP4B upregulation in cell growth, we analyzed cell proliferation, using a rescue assay in NPM1-mA silenced cells. The results showed that INPP4B protein levels were successfully recovered upon INPP4B overexpression
(Fig. 6c). Importantly, the inhibiting effect of NPM1-mA depletion-mediated cell proliferation was reversed by reexpression of INPP4B (Fig. 6d). These results indicate that INPP4B upregulation mediated by NPM1-mA promotes cell proliferation in OCI-AML3 cells.

\section{High expression of INPP4B is associated with poor survival outcome in NPM1-mutated leukemia} To investigate the function of INPP4B in AML patients with the NPM1 mutation, we investigated the prognostic 
a

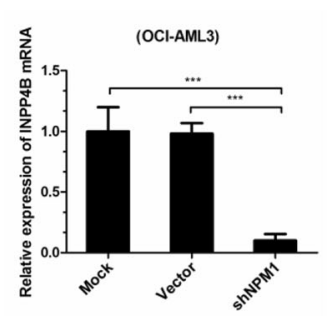

C

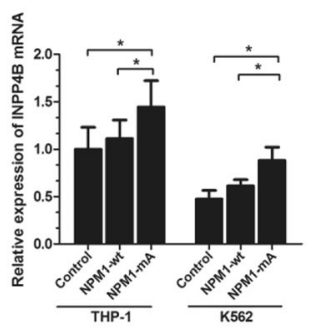

e

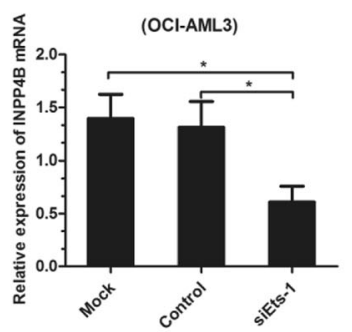

g

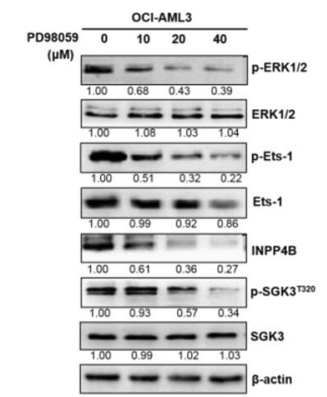

b

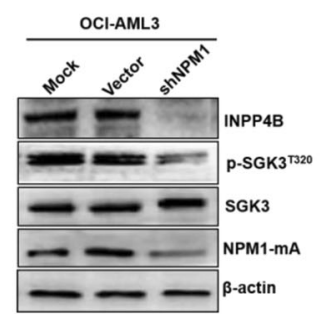

d

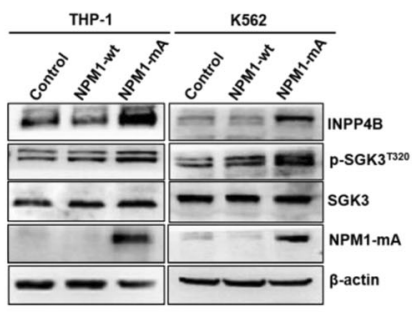

f

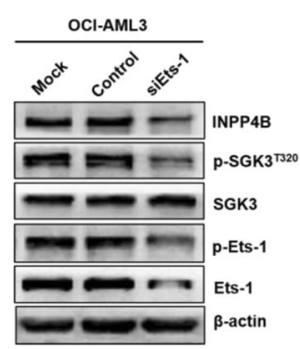

h

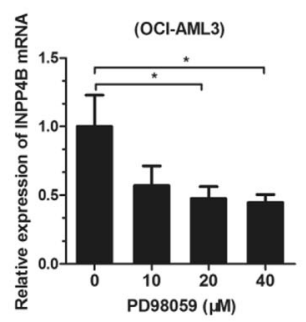

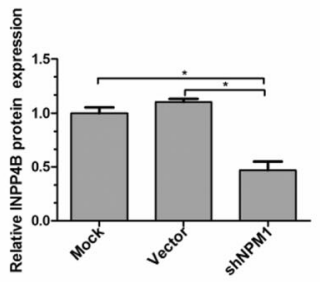
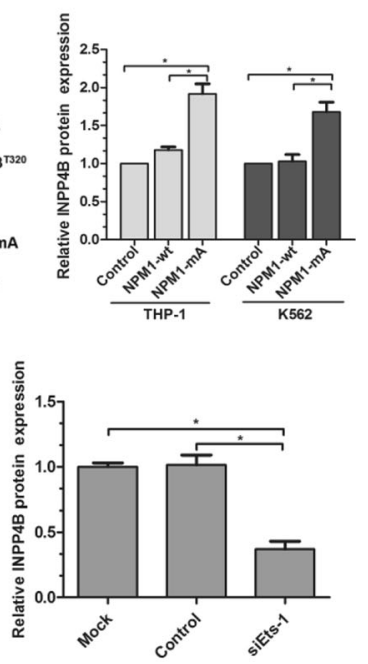

i

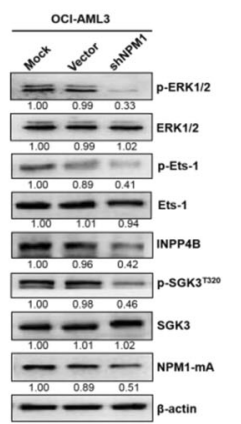

Fig. $\mathbf{5}$ INPP4B is upregulated by NPM1-mA in leukemia cells via ERK/Ets-1 signaling. (a) qRT-PCR analysis of INPP4B mRNA expression, (b) western blotting analysis of INPP4B, p-SGK3 ${ }^{\text {T320 }}$, SGK3 and NPM1-mA from the NPM1-mA-silenced OCI-AML3 cells. (c) qRT-PCR analysis of INPP4B mRNA expression, (d) western blotting analysis of INPP4B, p-SGK3 ${ }^{\text {T320 }}$, SGK3 and NPM1-mA from the THP-1 and K562 cells transduced with the plasmids expressing NPM1-wt or NPM1-mA. (e) qRT-PCR analysis of INPP4B mRNA expression, (f) western blotting analysis of INPP4B, p-SGK3 ${ }^{\text {T320, }}$ SGK3, p-Ets-1 and Ets-1 from the OCl-AML3 cells transfected with the control siRNA or siEts-1. (g) Western blotting analysis of p-ERK, ERK, p-Ets-1, Ets-1, INPP4B, p-SGK3 $3^{T 32}$ and SGK3, (h) qRT-PCR analysis of INPP4B mRNA expression from the OCI-AML3 cells treated with different concentration of PD98059 (0, 10, 20 and $40 \mu M)$. i Western blotting analysis of p-ERK, ERK, p-Ets-1, Ets-1, INPP4B, p-SGK3 ${ }^{\text {T320 }}$, SGK3 and NPM1-mA from the NPM1mA-silenced OCI-AML3 cells. Proteins were quantified using image software and normalized against $\beta$-actin. Data were represented as mean \pm s.d. of three individual experiments. ${ }^{*} p<0.05,{ }^{* *} p<0.001$

value of INPP4B using the dataset of TCGA, for which clinical data were available. First, Kaplan-Meier survival analysis was performed on 153 AML samples. The results showed that high INPP4B cohort had significant shorter OS (median, 14.3 vs 22.5 months; $p=0.02$, Fig. 7a) and the three-year EFS (median, 14.3 vs 22.5 months; $p=0.02$, Fig. 7b), compared with low INPP4B cohort of AML patients. Noticeably, we observed that NPM1-mutated patients with high INPP4B expression showed an approximate correlation with inferior survival (Fig. 7c). Furthermore, Kaplan-Meier survival analysis of 38 NPM1-mutated samples revealed that the OS (median, 10.1 vs 24.3 months; $p=0.04$, Fig. 7d) and the three-year EFS (median, 10.1 vs 24.3 months; $p=0.04$, Fig. 7e) was evidently shorter in high INPP4B cohort, as compared to the low INPP4B cohort. Moreover, the samples from TCGA confirmed the poor outcome (hazard ratio $[H R]=2.8$ ) of high 


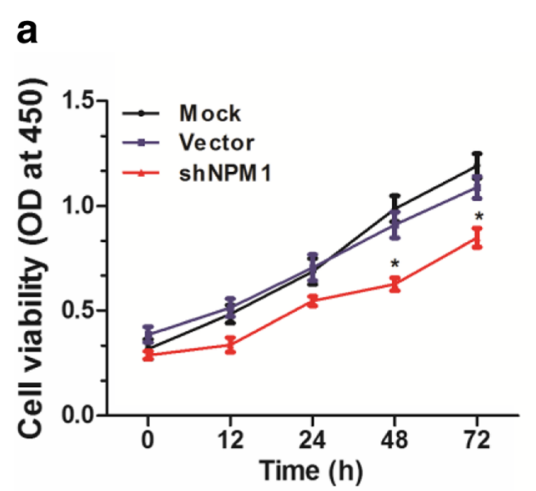

C

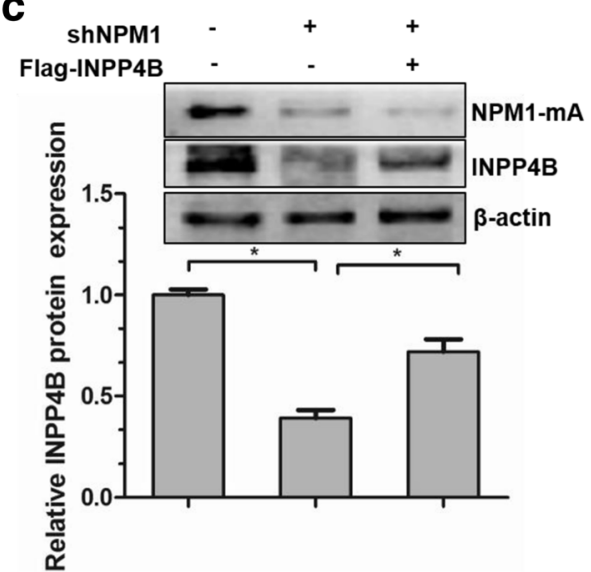

b

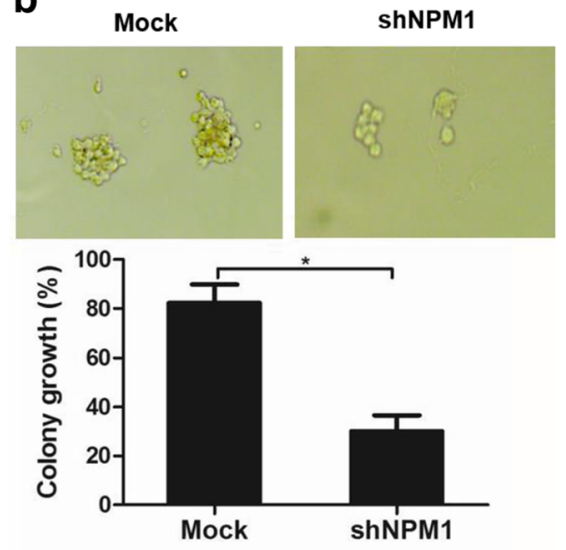

d

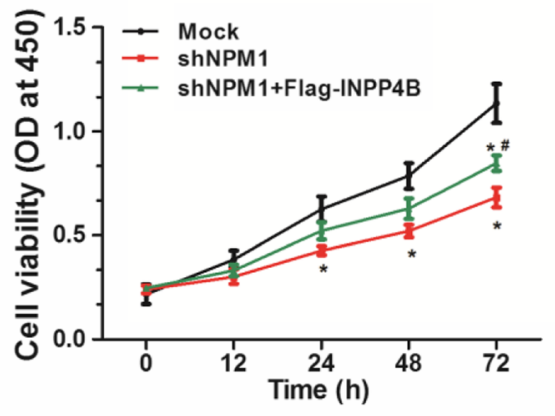

Fig. 6 NPM1-mA-mediated INPP4B upregulation promotes cell proliferation in OCI-AML3 cells. The NPM1-mA-silenced OCI-AML3 cells were subjected to (a) CCK8 assays and (b) colony forming assays. c The NPM1-mA-silenced OCl-AML3 cells were transfected with the pEAK-Flag/INPP4B plasmids, western blotting analysis of INPP4B and NPM1-mA. Proteins were quantified using image software and normalized against $\beta$-actin. $\mathbf{d}$ CCK-8 assay analysis of cell proliferation in NPM1-mA-silenced OCI-AML3 cells, followed by Flag-INPP4B introduction. Data were represented as mean \pm s.d. of three individual experiments. ${ }^{*} p<0.05$, as comparison to mock or vector group, respectively; $\# p<0.05$, as comparison to the shNPM1 group

INPP4B expression in NPM1-mutated AML. These clinical data from TCGA dataset indicate that high expression of INPP4B showed a trend towards poor prognosis in AML cases with NPM1 mutations.

\section{Discussion}

The NPM1 mutations are among the most frequent genetic alterations in AML, especially in cases with a normal karyotype. However, the pathogenesis of NPM1-mutated AML has not been fully elucidated. Herein, our data demonstrate that INPP4B functions to promote leukemia cell survival in a SGK3-dependent manner, high levels of INPP4B are at least partially caused by the NPM1 mutant via ERK/Ets-1 signaling, and high INPP4B is potentially correlated with poor clinical outcome in NPM1-mutated leukemia (Fig. 7f).

It has been known that INPP4B is a phosphoinositide phosphatase with pleiotropic functions in various cellular processes [36]. As a novel factor in the PI3K signaling pathway, INPP4B has been found to play a tumor suppressive role in prostate, breast, and ovarian cancers, and possibly in leukemia [37, 38]. However, unexpected findings from recent reports indicate that INPP4B function might be more complicated than previously thought [39]. In the present study, we analyzed INPP4B mRNA levels in NPM1-mutated AML patients from the dataset of TCGA and further detected INPP4B expression in NPM1-mutated AML primary blasts and NPM1-mA positive OCI-AML3 cells. These results revealed relative overexpression of INPP4B in NPM1-mutated AML. The overexpression of INPP4B first became evident after the analysis of gene expression in leukemic blasts from BCR/ABL-positive pediatric acute lymphoblastic leukemia [40]. High INPP4B expression was recently reported in a subset of AML patients with lower response rates to chemotherapy and shorter survival [41]. Next, we explored the biological effects of INPP4B overexpression in NPM1-mutated leukemia. We observed that knockdown of 


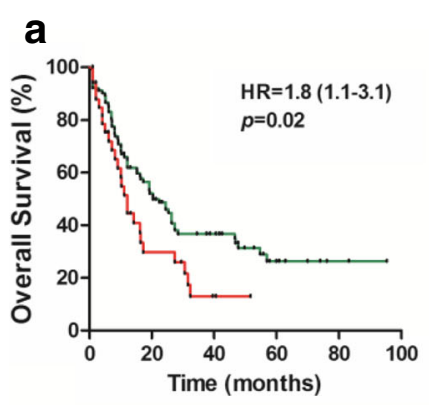

b

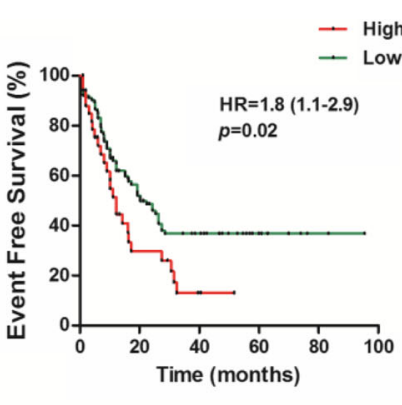

C
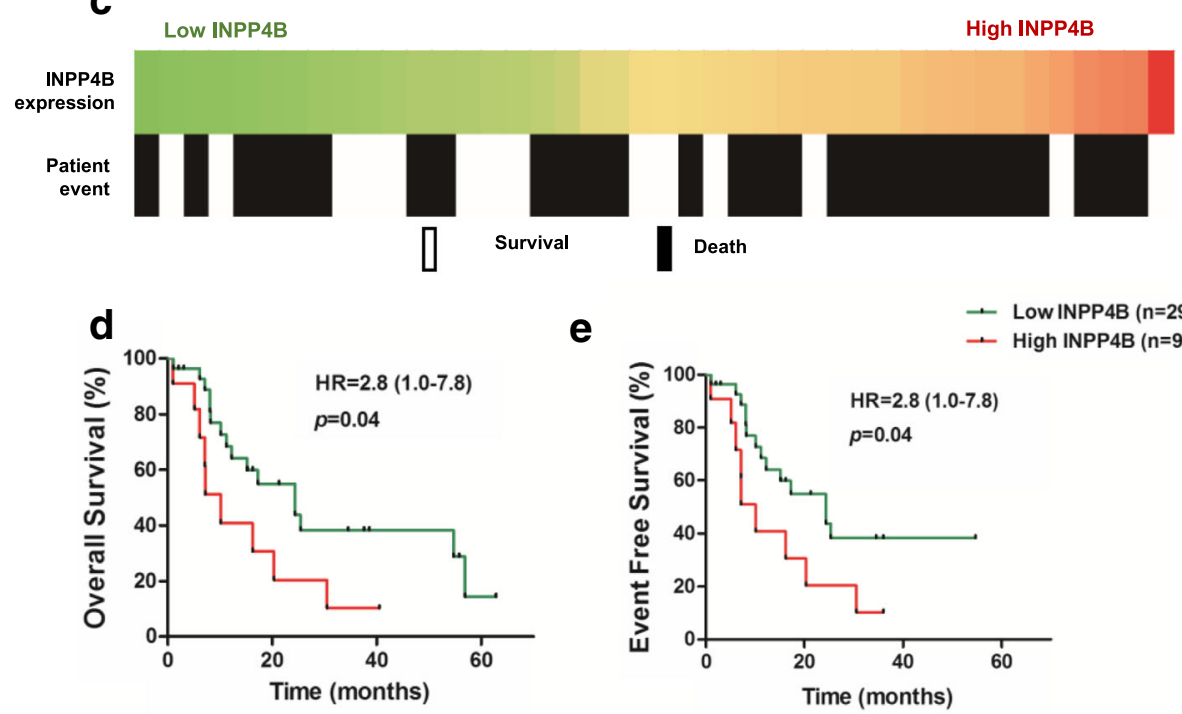

$\mathbf{f}$

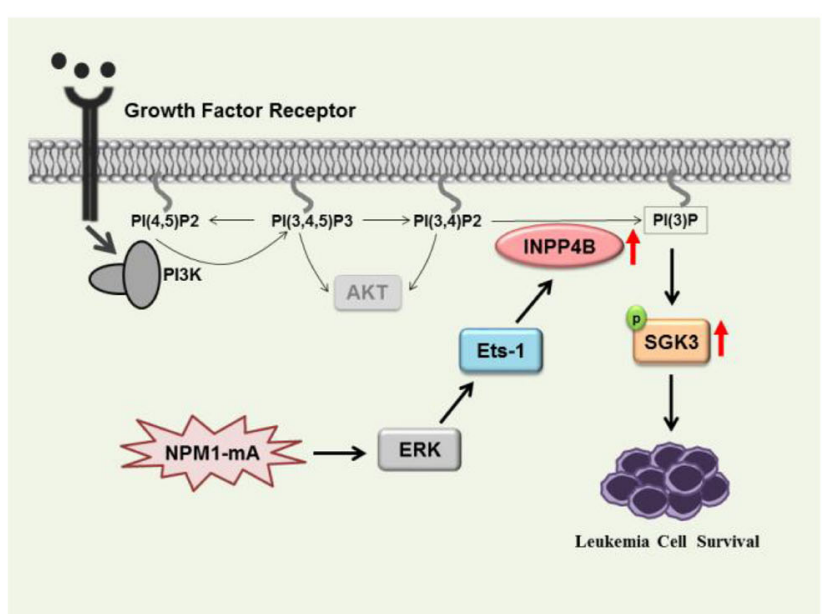

Fig. 7 High expression of INPP4B is associated with poor survival outcome in NPM1-mutated leukemia. Kaplan-Meier survival data of 153 AML patients were used to analysis (a) OS and (b) EFS curves according to INPP4B levels. c Heatmap of 38 primary AML samples with NPM1 mutation from TCGA dataset in which INPP4B expression was aligned with patient event (Survival or Death). Kaplan-Meier survival data of patients with NPM1-mutated AML were used to analysis (d) OS and (e) EFS curves according to INPP4B levels. $\mathbf{f}$ Schematic diagram describing the functional significance of INPP4B in the NPM1-mutated leukemia cells. INPP4B promotes leukemia cell survival in a SGK3-dependent and AKT-independent manner. The expression of INPP4B partially upregulated by NPM1-mA is due to ERK/Ets-1 signaling

INPP4B resulted in inhibition of OCI-AML3 cell proliferation in vitro, and conversely, recovered INPP4B could rescue this inhibitory effect, which confirms the gain-offunction of INPP4B in NPM1-mutated AML. Our findings were consistent with those of a previous study, in which reported that the introduction of INPP4B conferred a significant increase in the colony forming potential in OCIAML3 cells [41]. Moreover, Rijal et al. [25] reported that 
ectopic overexpression of INPP4B enhanced leukemic resistance to cytosine arabinoside (Ara-C). These findings and our data imply that the tumor suppressor gene INPP4B plays a potential oncogenic role in NPM1-mutated leukemia. In a future study, the function of INPP4B in mouse knock-in models that mimic human NPM1-mutated AML are worthy to be further investigated.

The INPP4B protein is a phosphoinositide phosphatase and acts as an important regulator in PI3K pathwayassociated cancer [42]. In the present study, we found that introduction of INPP4B elevated SGK3 phosphorylation and knockdown of INPP4B reduced SGK3 phosphorylation in OCI-AML3 cells. These results are consistent with those of another report, in which the expression of INPP4B leads to enhanced SGK3 activation in PIK3CAmutated breast cancer cells [23]. Because AKT is the canonical downstream effector of INPP4B in the PI3K pathway and INPP4B has the seemingly paradoxical role in AKT activation in a variety of different types of cancers [42], we tested whether INPP4B was involved in the regulation of phosphorylated AKT in leukemia cells. Surprisingly, our data demonstrate that loss or gain of INPP4B did not appear to affect AKT activation in NPM1-mutated leukemia cells. These results are consistent with those of a previous report of Rijal et al. [25], who reported no correlation between endogenous INPP4B protein levels and AKT phosphorylation status in AML. Next, we treated OCI-AML3 cells with PI3K pathway inhibitors and found that the PI3K inhibitor, LY294002 (but not the AKTselective inhibitor, MK-2206 and the mTOR inhibitor, rapamycin) markedly reduced the p-SGK3 levels. These findings and our data indicate that INPP4B mediates activation of the PI3K downstream factor, SGK3, but not AKT in NPM1-mutated leukemia. It is well known that INPP4B preferentially hydrolyzes PI $(3,4) \mathrm{P}_{2}$ to produce PI (3) $\mathrm{P}$ through its lipid phosphatase activity [39]. Importantly, SGK3 phosphorylation and subsequent activation are dependent on binding to the PI (3) P [43]. In our study, we observed that introduction of exogenous INPP4B increased PI (3) P levels in OCI-AML3 cell. These results reveal that enhanced activation of SGK3 mediated by INPP4B might be due to the accumulation of PI (3) P in NPM1mutated AML. Of note, INPP4B possesses both lipid and protein phosphatase activity [44], which remains possible that INPP4B also regulates phosphorylated SGK3 status through its protein phosphatase activity. Recently, Lopez et al. [44] has reported that K846M INPP4B mutant lacks lipid phosphatase activity but retains protein phosphatase activity. In the future study, we will further investigate the effects of INPP4B phosphatase activity on SGK3 using the K846M INPP4B mutant.
Considering the activation of SGK3 in NPM1-mutated leukemia cells, we assessed the role of SGK3 in INPP4Bmediated cell proliferation. The results from our experiments revealed that depletion of SGK3 led to significant inhibition of cell proliferation. We also performed a rescue assay and found that ectopic expression of SGK3 could reverse shINPP4B-induced inhibition of cell proliferation. Collectively, these findings support the hypothesis that INPP4B activates SGK3 signaling, to promote cell proliferation in NPM1-mutated leukemia. Several studies have demonstrated that SGK3 contributes to INPP4B-mediated cell proliferation in colon cancer [33] and a subset of melanomas cells [24]. In addition, INPP4B has been known to activate SGK3 and drive tumorigenesis in a subset of breast cancers with low levels of AKT [23]. Recently, a study has reported that INPP4B dephosphorylates tumor suppressor PTEN through its protein phosphatase activity and subsequent degradation of PTEN, thereby promotes cell proliferation of colon cancer [33]. Thus, in addition to SGK3, the other potential mechanisms underlying oncogenic role of INPP4B in NPM1mutated leukemia cells needs to be determined.

Because INPP4B is aberrantly expressed in NPM1mutated AML, the question is whether high expression of INPP4B in leukemic cells correlates with NPM1 mutations. We investigated the effects of NPM1 mutation on INPP4B expression. The results revealed that enforced expression of NPM1-mA increased INPP4B mRNA and protein levels, whereas knockdown of NPM1-mA had the opposite effect. As INPP4B protein expression seems largely correlated with its mRNA expression in NPM1mutated leukemia cells (Fig. 5a-d), it is likely that INPP4B is elevated by transcriptional mechanisms. We next searched for the specific transcription factors involved in this process. A recent paper has identified that the increased expression of INPP4B is due to transcriptional upregulation mediated by the transcription factor Ets- 1 in colon cancer cells [33]. In the present study, we found that siRNA-mediated knockdown of Ets-1 significantly downregulated INPP4B mRNA and protein levels in OCI-AML3 cells. It has been well documented that ERK activation is specifically required for the transcriptional function of Ets-1 [45]. Moreover, our previous study has verified that ERK signaling is continuously activated by NPM1-mA [35]. In this study, we treated OCI-AML3 cells with the ERK inhibitor, PD98059, and found that this treatment inhibited Ets-1 phosphorylation and further reduced the INPP4B levels in a dose-dependent manner. More notably, the loss of NPM1-mA weakened ERK/ Ets-1 signaling and then reduced INPP4B protein levels. These results indicate that INPP4B is partially upregulated by NPM1-mA via ERK/Ets1 signaling. Previous studies have reported that AML cases carrying NPM1 mutations are generally associated with other 
common mutations (FLT3-ITD or DNMT3A) [46, 47]. Recently, a study from TCGA Research Network showed the complex relationships of cooperation or mutual exclusivity among these mutations related to AML [48]. Future studies will determine whether there is any association between the presence of other mutations and the level of INPP4B in NPM1-mutated patients. In addition, NPM1 is a nucleolar phosphoprotein and its phosphorylation status alters its functions. Previous study has shown that NPM1 could be dephosphorylated on Thr199 by the Ser/Thr protein phosphatase PP1 $\beta$ in response to DNA damage [49]. Considering the fact that INPP4B has protein tyrosine phosphatase activity [44] and Ser/Thr phosphatase activity [33], whether NPM1 might be a substrate of the INPP4B protein in leukemia remains to be clarified.

Next, we also observed that knockdown of NPM1-mA significantly inhibited cell proliferation in OCI-AML3 cells and the introduction of INPP4B successfully reversed this inhibitory effect. These observations indicate that NPM1-mA enhanced INPP4B expression and promoted cell survival in AML. We have previously identified the crucial role of NPM1 mutations in the invasion phenotype [35], cell differentiation block [32], and autophagic activity [50] in AML. Finally, we evaluated the clinical significance of INPP4B in NPM1-mutated AML cases derived from the TCGA dataset. High levels of INPP4B delineated a poorer prognosis in AML patients. Importantly, NPM1-mutated patients with high INPP4B tended to have shorter survival outcome. Our results support this observation, by showing that high INPP4B levels are associated with a poor outcome in AML among six independent gene expression datasets [41].

\section{Conclusions}

In summary, for the first time to our knowledge, our data suggest that overexpression of INPP4B promotes NPM1mutated leukemia cell proliferation through SGK3 activation. High levels of INPP4B are at least partially induced by the NPM1 mutant via ERK/Ets-1 signaling. Importantly, high INPP4B showed a trend towards poor outcome in NPM1-mutated patients. These findings indicate that INPP4B targeting might be a potential therapeutic strategy for NPM1-mutated AML.

\footnotetext{
Abbreviations

AML: Acute myeloid leukemia; ATCC: American type culture collection; BM: Bone marrow; CCK8: Cell Counting Kit-8; Cdk2: Cyclin-dependent kinase 2; DNMT3A: DNA (cytosine-5-)-methyltransferase 3 alpha; DSMZ: Deutsche Sammlung von Mikroorganismen und Zellkulturen GmbH; EFS: Event free survival; ELISA: Enzyme-linked immunosorbent assay; ERK: Extracellular signal-regulated kinase; Ets-1: Erythroblastosis virus E26 oncogene homolog; FBS: Fetal bovine serum; FLT3-ITD: FMS-like tyrosine kinase 3-internal tandem duplication; HR: Hazard ratio; INPP4B: Inositol polyphosphate 4-phosphatase II; NK-AML: Normal karyotype acute myeloid leukemia; NPM1: Nucleophosmin; NPM1-mA: NPM1-mutation type A; NPM1-wt: Wildtype NPM1; OS: Overall survival; PI: Phosphoinositide; PI3K: Phosphoinositide3 kinase; PIP: Phosphatidylinositol phosphate; PIP2: Lipid phosphatidyl-
}

inositol bisphosphate; PIP3: Lipid phosphatidyl-inositol trisphosphate; PTEN: Phosphatase and tensin homolog; PVDF: Polyvinylidene difluoride; PX: Phox homology; qRT-PCR: Quantitative real-time PCR; RT-PCR: Reverse transcription; SDS: Sodium dodecyl sulfate; SGK3: Serum and glucocorticoidregulated kinase-3; SHIP: Src homology 2-containing inositol phosphatase; shRNA: Lentiviral small hairpin RNA; TBS: Tris-buffered saline; TCGA: The Cancer Genome Atlas; WHO: World Health Organization

\section{Acknowledgements}

The authors thank Dr. Falini B (Institute of Hematology, University of Perugia, Perugia, Italy) for providing the pEGFPC1-NPM1-mA, pEGFPC1-NPM1-wt and empty pEGFPC1 plasmids.

\section{Funding}

This work was supported by the National Natural Science Foundation of China (grant no. 81271913) and Graduate Fellowship in research innovation from the Chongqing Municipal Education Commission (grant no. CYS16140, CYS17155).

\section{Availability of data and materials}

Literature collection was performed using PubMed. Statistical analyses were executed by using SPSS 17.0 software (IBM, Chicago, IL, USA) and GraphPad (Prism 5.0). Raw and processed data are stored in corresponding author and are available upon request. The RNAseq datasets and clinical information of NPM1-mutated AMLs in the current study were retrieved from TCGA database (http://www.cancergenome.nih.gov).

\section{Authors' contributions}

$L Z$ and $H J J$ conceived and designed the experiments. HJJ, LYY and LZ performed the experiments and wrote the paper. ZLY and QZ prepared the patient samples. LW, YT and QZ collected and analyzed the data. YTT, JRX, SSZ and YPJ provided assistance with revising the manuscript. All authors read and approved the manuscript.

\section{Ethics approval and consent to participate}

All samples were collected with informed consent and the experiments were approved by the institutional ethics committee of Chongqing medical university and Third Military medical university.

\section{Consent for publication}

Not applicable.

Competing interests

All authors declare that they have no competing interests.

\section{Publisher's Note}

Springer Nature remains neutral with regard to jurisdictional claims in published maps and institutional affiliations.

\section{Author details}

${ }^{1}$ Key Laboratory of Laboratory Medical Diagnostics Designated by the Ministry of Education, School of Laboratory Medicine, Chongqing Medical University, Chongqing 400016, China. No.1, Yixueyuan Road, Chongqing 400016, China. ${ }^{2}$ Center for Hematology, Southwest Hospital, Third Military Medical University, Chongqing, China. ${ }^{3}$ The Center for Clinical Molecular Medical detection, The First Affiliated Hospital of Chongqing Medical University, Chongqing, China.

Received: 19 November 2017 Accepted: 4 January 2018

Published online: 17 January 2018

References

1. Papaemmanuil E, Gerstung M, Bullinger L, Gaidzik VI, Paschka P, Roberts ND, Potter NE, Heuser M, Thol F, Bolli N, et al. Genomic classification and prognosis in acute myeloid leukemia. N Engl J Med. 2016;374:2209-21.

2. Cancer Genome Atlas Research N, Ley TJ, Miller C, Ding L, Raphael BJ, Mungall AJ, Robertson A, Hoadley K, Triche TJ Jr, Laird PW, et al. Genomic and epigenomic landscapes of adult de novo acute myeloid leukemia. $\mathrm{N}$ Engl J Med. 2013;368:2059-74. 
3. Falini B, Gionfriddo I, Cecchetti F, Ballanti S, Pettirossi V, Martelli MP. Acute myeloid leukemia with mutated nucleophosmin (NPM1): any hope for a targeted therapy? Blood Rev. 2011;25:247-54.

4. Cagnetta A, Adamia S, Acharya C, Patrone F, Miglino M, Nencioni A, Gobbi M Cea M. Role of genotype-based approach in the clinical management of adult acute myeloid leukemia with normal cytogenetics. Leuk Res. 2014;38:649-59.

5. Falini B, Martelli MP. Impact of genomics in the clinical management of patients with cytogenetically normal acute myeloid leukemia. Best Pract Res Clin Haematol. 2015;28:90-7.

6. Falini B, Mecucci C, Tiacci E, Alcalay M, Rosati R, Pasqualucci L, La Starza R, Diverio D, Colombo E, Santucci A, et al. Cytoplasmic nucleophosmin in acute myelogenous leukemia with a normal karyotype. N Engl J Med. 2005; 352:254-66.

7. Falini B, Martelli MP, Bolli N, Bonasso R, Ghia E, Pallotta MT, Diverio D, Nicoletti I, Pacini R, Tabarrini A, et al. Immunohistochemistry predicts nucleophosmin (NPM) mutations in acute myeloid leukemia. Blood. 2006; 108:1999-2005.

8. Box JK, Paquet N, Adams MN, Boucher D, Bolderson E, O'Byrne KJ, Richard DJ. Nucleophosmin: from structure and function to disease development. BMC Mol Biol. 2016;17:19.

9. Arber DA, Orazi A, Hasseriian R, Thiele J, Borowitz MJ, Le Beau MM, Bloomfield CD, Cazzola M, Vardiman JW. The 2016 revision to the World Health Organization classification of myeloid neoplasms and acute leukemia. Blood. 2016;127:2391-405.

10. Falini B, Nicoletti I, Martelli MF, Mecucci C. Acute myeloid leukemia carrying cytoplasmic/mutated nucleophosmin (NPMc+ AML): biologic and clinical features. Blood. 2007;109:874-85.

11. Heath EM, Chan SM, Minden MD, Murphy T, Shlush LI, Schimmer AD. Biological and clinical consequences of NPM1 mutations in AML. Leukemia. 2017:31:798-807.

12. Park S, Chapuis N, Tamburini J, Bardet V, Cornillet-Lefebvre P, Willems L, Green A, Mayeux P, Lacombe C, Bouscary D. Role of the PI3K/AKT and mTOR signaling pathways in acute myeloid leukemia. Haematologica. 2010; 95:819-28

13. Fransecky L, Mochmann LH, Baldus CD. Outlook on PI3K/AKT/mTOR inhibition in acute leukemia. Mol Cell Ther. 2015:3:2.

14. Polak R, Buitenhuis M. The PI3K/PKB signaling module as key regulator of hematopoiesis: implications for therapeutic strategies in leukemia. Blood. 2012;119:911-23.

15. Faes S, Dormond O. PI3K and AKT: unfaithful Partners in Cancer. Int J Mol Sci. 2015;16:21138-52.

16. Chalhoub N, Baker SJ. PTEN and the PI3-kinase pathway in cancer. Annu Rev Pathol. 2009:4:127-50.

17. Eramo MJ, Mitchell CA. Regulation of Ptdlns $(3,4,5)$ P3/Akt signalling by inositol polyphosphate 5-phosphatases. Biochem Soc Trans. 2016:44:240-52.

18. Gewinner C, Wang ZC, Richardson A, Teruya-Feldstein J, Etemadmoghadam D, Bowtell D, Barretina J, Lin WM, Rameh L, Salmena L, et al. Evidence that inositol polyphosphate 4-phosphatase type II is a tumor suppressor that inhibits PI3K signaling. Cancer Cell. 2009:16:115-25.

19. Vo TT, Fruman DA. INPP4B is a tumor suppressor in the context of PTEN deficiency. Cancer Discov. 2015:5:697-700.

20. Fedele CG, Ooms LM, Ho M, Vieusseux J, O'Toole SA, Millar EK, LopezKnowles E, Sriratana A, Gurung R, Baglietto L, et al. Inositol polyphosphate 4-phosphatase II regulates PI3K/Akt signaling and is lost in human basal-like breast cancers. Proc Natl Acad Sci U S A. 2010;107:22231-6.

21. Balakrishnan A, Chaillet JR. Role of the inositol polyphosphate-4 phosphatase type II Inpp4b in the generation of ovarian teratomas. Dev Biol. 2013;373:118-29.

22. Hodgson MC, Shao L, Frolov A, Li R, Peterson LE, Ayala G, Ittmann MM, Weigel $\mathrm{NL}$, Agoulnik IU. Decreased expression and androgen regulation of the tumor suppressor gene INPP4B in prostate cancer. Cancer Res. 2011;71:572-82.

23. Gasser JA, Inuzuka H, Lau AW, Wei W, Beroukhim R, Toker A. SGK3 mediates INPP4B-dependent PI3K signaling in breast cancer. Mol Cell. 2014:56:595-607.

24. Chi MN, Guo ST, Wilmott JS, Guo XY, Yan XG, Wang CY, Liu XY, Jin L, Tseng HY, Liu T, et al. INPP4B is upregulated and functions as an oncogenic driver through SGK3 in a subset of melanomas. Oncotarget. 2015:6:39891-907.

25. Rijal S, Fleming S, Cummings N, Rynkiewicz NK, Ooms LM, Nguyen NY, Teh TC, Avery S, McManus JF, Papenfuss AT, et al. Inositol polyphosphate 4phosphatase II (INPP4B) is associated with chemoresistance and poor outcome in AML. Blood. 2015;125:2815-24.
26. Bruhn MA, Pearson RB, Hannan RD, Sheppard KE. Second AKT: the rise of SGK in cancer signalling. Growth Factors. 2010;28:394-408.

27. Lien EC, Dibble CC, Toker A. PI3K signaling in cancer: beyond AKT. Curr Opin Cell Biol. 2017:45:62-71.

28. Tessier M, Woodgett JR. Role of the Phox homology domain and phosphorylation in activation of serum and glucocorticoid-regulated kinase3. J Biol Chem. 2006;281:23978-89.

29. Vasudevan KM, Barbie DA, Davies MA, Rabinovsky R, McNear CJ, Kim JJ, Hennessy BT, Tseng H, Pochanard P, Kim SY, et al. AKT-independent signaling downstream of oncogenic PIK3CA mutations in human cancer. Cancer Cell. 2009;16:21-32.

30. Quentmeier H, Martelli MP, Dirks WG, Bolli N, Liso A, Macleod RA, Nicoletti I, Mannucci R, Pucciarini A, Bigerna B, et al. Cell line OCI/AML3 bears exon-12 NPM gene mutation-a and cytoplasmic expression of nucleophosmin. Leukemia. 2005:19:1760-7.

31. Bruhn MA, Pearson RB, Hannan RD, Sheppard KE. AKT-independent PI3-K signaling in cancer - emerging role for SGK3. Cancer Manag Res. 2013;5:281-92

32. Zou Q, Tan S, Yang Z, Wang J, Xian J, Zhang S, Jin H, Yang L, Wang L, Zhang L. The human nucleophosmin 1 mutation a inhibits myeloid differentiation of leukemia cells by modulating miR-10b. Oncotarget. 2016;7:71477-90.

33. Guo ST, Chi MN, Yang RH, Guo XY, Zan LK, Wang CY, Xi YF, Jin L, Croft A, Tseng HY, et al. INPP4B is an oncogenic regulator in human colon cancer. Oncogene. 2016;35:3049-61.

34. Foulds CE, Nelson ML, Blaszczak AG, Graves BJ. Ras/mitogen-activated protein kinase signaling activates Ets-1 and Ets-2 by CBP/p300 recruitment. Mol Cell Biol. 2004:24:10954-64.

35. Xian J, Shao H, Chen X, Zhang S, Quan J, Zou Q, Jin H, Zhang L. Nucleophosmin mutants promote adhesion, migration and invasion of human leukemia THP-1 cells through MMPs up-regulation via Ras/ERK MAPK signaling. Int J Biol Sci. 2016;12:144-55.

36. Li H, Marshall AJ. Phosphatidylinositol $(3,4)$ bisphosphate-specific phosphatases and effector proteins: a distinct branch of PI3K signaling. Cell Signal. 2015:27:1789-98.

37. Agoulnik IU, Hodgson MC, Bowden WA, Ittmann MM. INPP4B: the new kid on the PI3K block. Oncotarget. 2011:2:321-8.

38. Kofuji S, Kimura H, Nakanishi H, Nanjo H, Takasuga S, Liu H, Eguchi S, Nakamura $\mathrm{R}$, Itoh $\mathrm{R}$, Ueno $\mathrm{N}$, et al. INPP4B is a Ptdlns $(3,4,5)$ P3 Phosphatase that can act as a tumor suppressor. Cancer Discov. 2015;5:730-9.

39. Woolley JF, Dzneladze I, Salmena L. Phosphoinositide signaling in cancer: INPP4B Akt (s) out. Trends Mol Med. 2015:21:530-2.

40. Ross ME, Zhou X, Song G, Shurtleff SA, Girtman K, Williams WK, Liu HC, Mahfouz R, Raimondi SC, Lenny N, et al. Classification of pediatric acute lymphoblastic leukemia by gene expression profiling. Blood. 2003;102:2951-9.

41. Dzneladze I, He R, Woolley JF, Son MH, Sharobim MH, Greenberg SA, Gabra M, Langlois C, Rashid A, Hakem A, et al. INPP4B overexpression is associated with poor clinical outcome and therapy resistance in acute myeloid leukemia. Leukemia. 2015;29:1485-95.

42. Rodgers SJ, Ferguson DT, Mitchell CA, Ooms LM. Regulation of PI3K effector signalling in cancer by the phosphoinositide phosphatases. Biosci Rep. 2017;37

43. Moniz LS, Vanhaesebroeck B. AKT-ing out: SGK kinases come to the fore. Biochem J. 2013;452:e11-3.

44. Lopez SM, Hodgson MC, Packianathan C, Bingol-Ozakpinar O, Uras F, Rosen BP Agoulnik IU. Determinants of the tumor suppressor INPP4B protein and lipid phosphatase activities. Biochem Biophys Res Commun. 2013:440:277-82.

45. Plotnik JP, Budka JA, Ferris MW, Hollenhorst PC. ETS1 is a genome-wide effector of RAS/ERK signaling in epithelial cells. Nucleic Acids Res. 2014;42:11928-40.

46. Falini B, Nicoletti I, Bolli N, Martelli MP, Liso A, Gorello P, Mandelli F, Mecucci C, Martelli MF. Translocations and mutations involving the nucleophosmin (NPM1) gene in lymphomas and leukemias. Haematologica. 2007:92:519-32.

47. Ley TJ, Ding L, Walter MJ, McLellan MD, Lamprecht T, Larson DE, Kandoth C, Payton JE, Baty J, Welch J, et al. DNMT3A mutations in acute myeloid leukemia. N Engl J Med. 2010;363:2424-33.

48. Li Y, Zhu B. Acute myeloid leukemia with DNMT3A mutations. Leuk Lymphoma. 2014:55:2002-12

49. Lin CY, Tan BC, Liu H, Shih CJ, Chien KY, Lin CL, Yung BY. Dephosphorylation of nucleophosmin by PP1 beta facilitates pRB binding and consequent E2F1dependent DNA repair. Mol Biol Cell. 2010;21:4409-17.

50. Zou Q, Tan S, Yang Z, Zhan Q, Jin H, Xian J, Zhang S, Yang L, Wang L, Zhang L. NPM1 mutant mediated PML delocalization and stabilization enhances Autophagy and cell survival in leukemic cells. Theranostics. 2017; 7:2289-304. 